\title{
Resting-state alpha rhythm modulation after divergent problem solving in aged adults
}

\author{
Evgeniya Privodnova \\ Scientific Research Institute of \\ Physiology and Basic Medicine \\ Novosibirsk, Russia \\ privodnovaeu@physiol.ru
}

\author{
Nina Volf \\ Scientific Research Institute of \\ Physiology and Basic Medicine \\ Novosibirsk, Russia volf@physiol.ru \\ Victoriya Bilik \\ Scientific Research Institute of \\ Physiology and Basic Medicine \\ Novosibirsk, Russia v.bilik@mail.ru
}

\author{
Ekaterina Merculova \\ Scientific Research Institute of \\ Physiology and Basic Medicine \\ Novosibirsk, Russia \\ merkaterine@gmail.com
}

\begin{abstract}
Given that repetitive cognitive activity leaves posttask traces after task performance and divergent problem solving is accompanied by alpha power enhancement, one may suppose that divergent tasks performance may induce resting-state alpha rhythm modulation. The results obtained in the current pilot study indicated that alpha power increased after 30-40 minute divergent problem solving in parietal brain region in older adults.
\end{abstract} aging

Keywords - alpha rhythm modulation, divergent thinking,

\section{Motivation and aim}

\section{Motivation}

Resting-state EEG alpha power is known to dramatically decrease as people ages [1]. At the same time, due to the association between alpha power and inhibitory abilities [2, 3], alpha increase may be useful to preserve top-down inhibitory capacity in older age. Given that repetitive cognitive activity leaves post-task traces after task performance [4] and that divergent problem solving is accompanied by alpha power enhancement [5], one may suppose that divergent tasks performance may results in resting-state alpha rhythm modulation.

Aim

The current study aimed to examine if alpha power increases after performance of verbal and visual divergent tasks. To this end, we explored the changes in resting-state alpha power that followed 30-40 minute divergent problem solving session, which consists of repetitive tasks of verbal and visual domains.

\section{Methods}

We recorded EEG in 30 right handed older adults (55-75 years old, mean age $=64.2$ ) before, during and after divergent thinking session. All subjects continued their professional activity; exclusion criteria was self-reported history of psychiatric, major medical, and neurological diseases. Divergent thinking session lasted for 30-40 minutes and included 30 verbal tasks ("Alternate Uses Task", [6]) and 30 visual tasks ("Incomplete figures", [6]). Originality (uniqueness) of creative ideas in each creativity domain was assessed using database of the response rate in these tests developed by authors previously. The EEG data were registered using $52 \mathrm{Ag}-\mathrm{AgCl}$ electrodes placed according to the modified version of the international 10 20 system via "Neuroscan 4.4" (USA). Fronto-central electrode was used as the ground, and electronically linked mastoid electrodes as reference. Electrode impedances did not exceed 5 $\mathrm{k} \Omega$. The EEG was amplified using Neuroscan amplifiers with a gain of 250 and a bandpass of $0-50 \mathrm{~Hz}$. Artifacts rejection was made using independent component analysis implemented in EEGLAB toolbox (https://sccn.ucsd.edu/eeglab/). Bandwidth for the upper alpha band was defined as [(individual alpha peak frequency) to (individual alpha peak frequency +2)]. To examine alpha activity, we used three-minute recordings in eyesopen resting-state condition from pre-test and post-test intervals as well as EEG recorded during successfully resolved verbal and visual tasks. In all cases, EEG data were segmented into $2 \mathrm{~s}$ epochs and submitted to further analysis. We calculated current source density (CSD) estimates via standardized Low Resolution Brain Electromagnetic Tomography (sLORETA) [13]. For each interval under analysis, we yielded the CSD distribution across all 6239 voxels in a realistic head model, which is based on the MNI 152 template, with spatial resolution of $5 \times 5 \times 5 \mathrm{~mm}$. Statistical contrasts of CSD estimates was performed using statistical nonparametric mapping (SnPM) with 5000 randomizations in the LORETA package. Pearson correlations were calculated in STATISTICA10. MNI coordinates of voxel with maximum difference between pre-test CSD and post-test CSD was used as a "seed" in follow-up region of interest (ROI) analysis. ROI was identified as cluster of voxels within the circle centered at seed, $15 \mathrm{~mm}$ in radius. For ROI analysis, eLORETA transformation matrix was used. ROIs were extracted from differential maps of post-task/pre-task changes, visual/pre-task changes, verbal/pre-task changes.

\section{Results}

Alpha CSD increased from pre-task to post-task resting-state interval $(\mathrm{p}=0.019)$ in the Superior Parietal lobule (Brodmann area 7) (see Figure, A). CSD estimates during visual task were higher in comparison with CSD estimates in pre-task interval with localization of maximal difference $(p=0.0003)$ in the Superior Parietal lobule (Brodmann area 7). However, CSD estimates during verbal task did not differ from CSD estimates in pre-task interval. The results suggest that alpha increase from pre-task to post-task interval is more likely to be formed by activity during performance of visual task. Correlation analysis of CSD estimates within ROIs revealed positive association between visual task and post-task intervals $(\mathrm{r}=0.46, \mathrm{p}=0.013$ ) (see Figure, B), while no significant correlations were found between verbal task and post-task intervals, providing additional evidence for the prominent role of visual domain. ROI estimates extracted from visual/pre-task changes were positively correlated with visual originality $(r=0.48, p=0.008)$ (see Figure, C). This finding suggests that CSD estimates of visual/pre-task changes within ROI reflected specific to visual creativity aspects of task performance. Correlations between efficacy indicator and CSD estimates in ROI, derived from post-task/pre-task changes, failed to reach significance level. Therefore, the traces 
in post-task EEG may be associated with extensive use of this region during visual task implementation, independently of efficiency of task performance.

\section{ACKNOWLEDGMENT}

Supported by budgetary funding for basic scientific research (theme No. AAAA-A16-116021010228-0, EEG data collection) and by RFBR and Government of the Novosibirsk region according to the research project No. 19-415-543009 (EEG data processing and analysis, conceptualization, paper writing and preparation).

\section{REFERENCES}

[1] R. J. Vaden, N. L. Hutcheson, L. A. McCollum, J. Kentros, and K. M. Visscher, "Older adults, unlike younger adults, do not modulate alpha power to suppress irrelevant information", Neuroimage, vol. 63(3), pp. 1127-1133, Aug. 2012.

[2] O. Jensen and A. Mazaheri, "Shaping functional architecture by oscillatory alpha activity: gating by inhibition", Front. Hum. Neurosci., vol. 4:e186, Nov. 2010.

[3] G. Borghini, M. Candini, C. Filannino, M. Hussain, V. Walsh, V. Rome, N. Zokaei, and M. Cappelletti, "Alpha Oscillations Are Causally Linked to Inhibitory Abilities in Ageing” J. Neurosci. vol. 38 (18), pp. 4418-4429, May 2018.

[4] C. Moisello, H.B. Meziane, S. Kelly, B. Perfetti, S. Kvint, N. Voutsinas, D. Blanco, A. Quartarone, G. Tononi and M.F. Ghilardi, "Neural activations during visual sequence learning leave a trace in post-training spontaneous EEG”, PloS one, vol. 8(6):e65882, Jun 2013.

[5] Fink and M. Benedek, "EEG alpha power and creative ideation", Neurosci. Biobehav. Rev., vol. 44, pp. 111-123, Jul. 2014.

[6] E.P. Torrance, "Torrance tests of creative thinking norms-technical manual : verbal tests, forms A and B : figural tests, forms A and B", Princeton, New Jersey Personal Press, 1966.

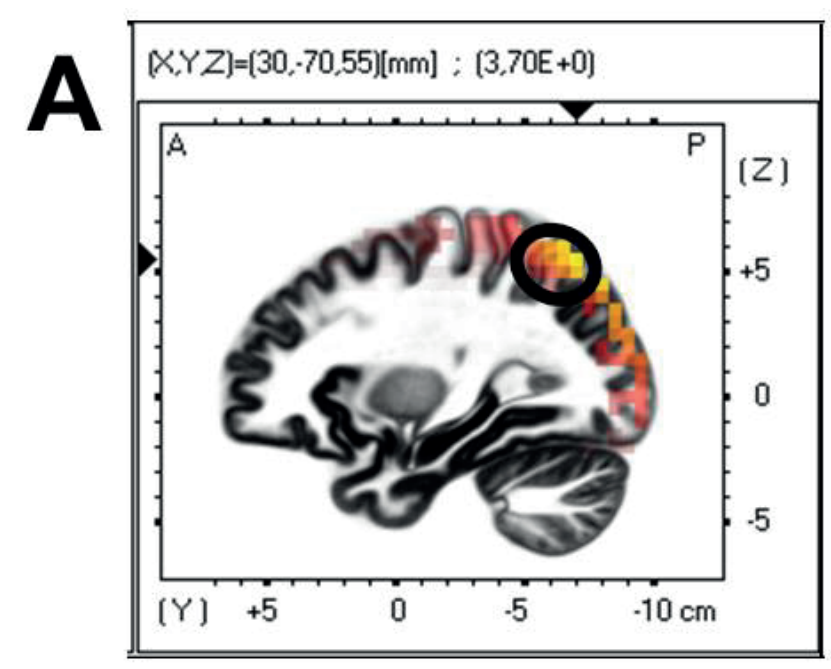

B

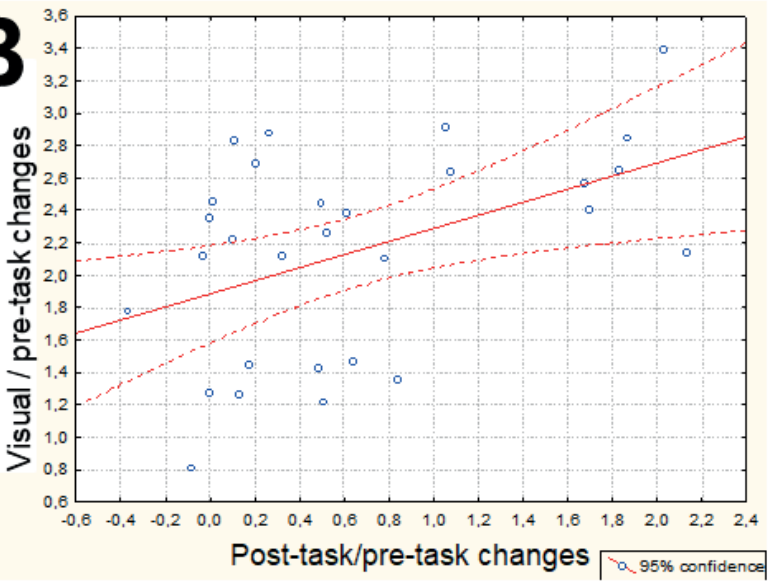

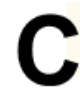

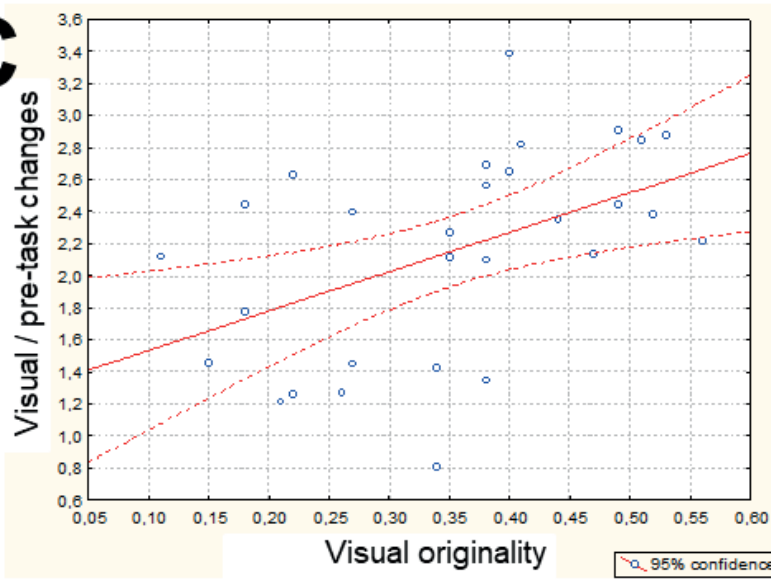

Fig. 1. Associations of CSD estimates within ROI in upper-alpha frequency band. A) Increase in CSD estimates from pre-task to post-task interval was statistically significant according to sLORETA SnPM in right Superior Parietal Lobule (Brodmann area 7). B) Correlations between ROI estimates of post-task/pre-task changes and ROI estimates of visual/pre-task changes. C) Correlations between ROI estimates of visual/pre-task changes with originality in visual task. Significant differences $(\mathrm{p}<0.05)$ are colored. ROI is marked by circle 